\title{
Therapy-Related Acute Myeloid Leukemia Diagnosed at Postmortem in a Long-term Survivor of Advanced Hepatocellular Carcinoma
}

Ki Rim Lee ${ }^{1}$, Jin Ho Paik ${ }^{1}$, Soong Deok Lee ${ }^{2,3}$, Moon-Young $\mathrm{Kim}^{4}$

${ }^{1}$ Department of Pathology, Seoul National University Bundang Hospital, Seoul National University College of Medicine, Seongnam, Korea, ${ }^{2}$ Department of Forensic Medicine, Seoul National University College of Medicine, Seoul, Korea, ${ }^{3}$ Institute of Forensic and Anthropological Science, Seoul National University College of Medicine, Seoul, Korea, ${ }^{4}$ Department of Anatomy and Cell Biology, Sungkyunkwan University School of Medicine, Suwon, Korea

Received: September 2, 2021 Revised: September 17, 2021 Accepted: November 15, 2021

\section{Correspondence to}

Moon-Young Kim

Department of Anatomy and Cell Biology, Sungkyunkwan University School of Medicine, 2066 Seobu-ro, Jangan-gu, Suwon 16419, Korea Tel: +82-31-299-6077

Fax: +82-31-299-6029

E-mail: tttrack@naver.com
Acute myeloid leukemia (AML) is a common hematologic malignancy with high mortality and a short survival period in adults. About $10 \%$ of these cases, called therapy-related AML, are reported to be the consequence of chemotherapy or radiotherapy of previous malignancy. In a clinical setting, this is usually diagnosed by peripheral blood smear or bone marrow biopsy by assessing the proportion of blasts. However, postmortem blood samples are not suitable for smear analysis because of hemolysis. Therefore, ancillary tests for identifying leukemic infiltration or related molecular change can provide an alternative diagnostic clue for AML. The deceased had been treated for 3 years for a combined type of hepatocellular carcinoma with multiple pulmonary metastases. Treatments included the resections of primary and metastatic tumors, chemotherapy, and radiotherapy, which prevented further progression of his cancer. One year after the last treatment, he suddenly collapsed without any specific symptoms and shortly died. The microscopic examination of the autopsy samples revealed extensive extramedullary infiltration of leukemia, which was confirmed as an AML by a series of ancillary immunohistochemical staining. This case illustrates both the importance of careful hematologic observation in cancer survivors and the necessity of a detailed medical diagnosis in a medicolegal autopsy.

Key Words: Acute myeloid leukemia; Leukemic infiltration; Treatment-related neoplasm; Hepatocellular carcinoma; Autopsy; Postmortem diagnosis

\section{Introduction}

Acute myeloid leukemia (AML) is the most common type of adult leukemia and accounts for the highest percentage of leukemic deaths [1,2]. This can rapidly progress in cases of monocytic differentiation, black race, older age at diagnosis, or less intensive treatment [3]. Most cases appear as de novo malignancy in previously healthy people. However, a subset of AML can arise from previous chemotherapy or radiotherapy, 
called therapy-related AML ( $\mathrm{t}-\mathrm{AML}$ ). These comprise about $7 \%-15 \%$ of all AML cases $[1,4-8]$.

Although infrequent, leukemic cell infiltrations into extramedullary tissues is one characteristic manifestation of AML [2]. This causes systemic symptoms or a multiorgan failure. This can provide a diagnostic clue at autopsy in specific circumstances, for example, when antemortem blood or bone marrow samples were not available because of rapid collapse or out-of-hospital death.

Here we report on an autopsy case of unexpected AML in a patient who had received serial chemotherapy and radiotherapy for metastatic hepatocellular carcinomas
(HCCs). We aim to emphasize the importance of careful hematologic observation in cancer survivors and a detailed diagnosis in a medicolegal autopsy.

\section{Case Report}

A 52-year-old male was found unconscious in front of his apartment. He had no response to resuscitation and expired after 3 hours. A blood test at the emergency room (Table 1) showed an elevated proportion of monocytes and about a ten-fold increase of the total number of white blood cells (WBCs). However, the doctors could not acquire his bone marrow sample

Table 1. The results of the antemortem blood tests of the deceased

\begin{tabular}{|c|c|c|c|c|}
\hline \multirow{2}{*}{ Items } & \multicolumn{2}{|l|}{ The day of death } & \multicolumn{2}{|c|}{6 months before death } \\
\hline & Results (reference range) & Status & Results (reference range) & Status \\
\hline Total WBC count $(/ \mu \mathrm{L})$ & $104,040(3,700-9,800)$ & $\uparrow$ & $3,580(4,000-10,000)$ & $\downarrow$ \\
\hline Neutrophils (\%) & $38.7(38.1-71.5)$ & - & $27(50-75)$ & $\downarrow$ \\
\hline Eosinophils (\%) & $6.6(0.5-8.6)$ & - & $3(1-5)$ & - \\
\hline Basophils (\%) & $0.1(0.1-1.1)$ & - & $0(0-2)$ & - \\
\hline Lymphocytes (\%) & $5.8(20.3-50.8)$ & $\downarrow$ & $53(20-44)$ & $\uparrow$ \\
\hline Monocytes (\%) & $48.8(3.5-9.9)$ & $\uparrow$ & $16(2-9)$ & $\uparrow$ \\
\hline Total RBC count $(/ \mu \mathrm{L})$ & $870(4,340-5,700)$ & $\downarrow$ & $2,980(4,200-6,300)$ & $\downarrow$ \\
\hline Hemoglobin (g/dL) & $3.7(14.0-17.6)$ & $\downarrow$ & $11.1(13-17)$ & $\downarrow$ \\
\hline Platelet count $(/ \mu \mathrm{L})$ & $40,000(160,000-362,000)$ & $\downarrow$ & $149,000(130,000-400,000)$ & - \\
\hline Serum $\mathrm{Na}^{+}(\mathrm{mmol} / \mathrm{L})$ & $131(135-145)$ & $\downarrow$ & $\mathrm{N} / \mathrm{A}$ & - \\
\hline Serum $\mathrm{K}^{+}(\mathrm{mmol} / \mathrm{L})$ & $6.5(3.5-5.5)$ & $\uparrow$ & $\mathrm{N} / \mathrm{A}$ & - \\
\hline Serum P (mg/dL) & $10.8(2.8-4.5)$ & $\uparrow$ & $3.5(2.5-4.5)$ & - \\
\hline Serum uric acid (mg/dL) & $13.6(2.8-8.2)$ & $\uparrow$ & $7.0(3.0-7.0)$ & - \\
\hline BUN (mg/dL) & $50.5(8-20)$ & $\uparrow$ & $32(10-26)$ & $\uparrow$ \\
\hline Serum creatinine (mg/dL) & $3.96(0.7-1.2)$ & $\uparrow$ & $1.47(0.7-1.4)$ & $\uparrow$ \\
\hline AST (IU/L) & $905(16-49)$ & $\uparrow$ & $22(1-40)$ & - \\
\hline ALT (IU/L) & $852(10-40)$ & $\uparrow$ & $26(1-40)$ & - \\
\hline Y-GTP (IU/L) & $43(10-90)$ & - & $28(11-63)$ & - \\
\hline Serum total bilirubin (mg/dL) & $2.24(0.3-1.9)$ & $\uparrow$ & $0.5(0.2-1.2)$ & - \\
\hline LDH (IU/L) & $>1,738(0-250)$ & $\uparrow$ & $\mathrm{N} / \mathrm{A}$ & - \\
\hline Blood ammonia ( $\mu \mathrm{g} / \mathrm{dL})$ & $1,097(27-102)$ & $\uparrow$ & $\mathrm{N} / \mathrm{A}$ & - \\
\hline Serum CK-MB (ng/mL) & $11.15(0-5.20)$ & $\uparrow$ & $\mathrm{N} / \mathrm{A}$ & - \\
\hline Serum troponin-I (pg/mL) & $88.16(0-26.20)$ & $\uparrow$ & $\mathrm{N} / \mathrm{A}$ & - \\
\hline Serum pro-BNP (pg/mL) & $2,687.7(0-273.5)$ & $\uparrow$ & N/A & - \\
\hline Serum CRP (mg/dL) & $4.91(0-0.6)$ & $\uparrow$ & $\mathrm{N} / \mathrm{A}$ & - \\
\hline
\end{tabular}

Reference ranges differ because of differences between the hospitals where the deceased had visited for a hepatocellular carcinoma follow-up and where he was transferred for resuscitation.

WBC, white blood cell; RBC, red blood cell; BUN, blood urea nitrogen; AST, aspartate transaminase; ALT, alanine transaminase; $\gamma$-GTP, $\gamma$-glutamyl transpeptidase; $\mathrm{LDH}$, lactate dehydrogenase; CK-MB, creatine kinase-myocardial band; BNP, brain natriuretic peptide; CRP, C-reactive protein. 
because of continuous resuscitation. There was no ST-elevation on the electrocardiogram, and the echocardiogram demonstrated dilated right ventricle and a D-shaped left ventricle. His family stated that he had fatigue, fever, back pain, and dizziness for 5 days before death and had been treated for HCC and its pulmonary metastases.

An autopsy was conducted after 36 hours to clear the cause and manner of his death. The height and weight were $172 \mathrm{~cm}$ and $90 \mathrm{~kg}$. There was a reversed L-shape scar on the right upper abdomen. A compensatory hyperplasia of the remaining liver $(1,894 \mathrm{~g})$ and splenomegaly $(846 \mathrm{~g})$ were observed. Several fibrotic lesions were found in both lungs, which seemed to be radiologic or surgical scars. The heart (486 g) showed prominent petechiae on the epicardium and coronary atherosclerosis with focal narrowing up to $90 \%$, however, no ischemic lesion was identified at the myocardium. There was no other grossly visible lesion such as mass, inflammation, or injury in all major organs investigated. Other pathologic signs, such as fluid accumulation, hemorrhage, or edema, were not recognized in the whole body.

In the peripheral blood smear (PBS), the proportion of WBCs was increased, and large atypical mononuclear cells were frequently observed despite postmortem hemolysis (Fig. 1A, B). On microscopic examination of fixed tissues, neoplastic hematologic cells had infiltrated the microvasculature and the interstitium of the major organs, including the heart, lungs, liver, and kidneys (Fig. 1C-F). Some large monocytes with eosinophilic cytoplasm were morphologically consistent with blasts.

Several days after the autopsy and funeral, an additional medical records were provided. Four years previously, on a background of hepatitis B virus-related cirrhosis, he had been diagnosed with HCC with a $10 \%$ cholangiocarcinoma component following left hemihepatectomy. He had localized radiotherapy for right pulmonary metastases right after the surgery. Then, a total of 28 cycles of systemic chemotherapies with gemcitabine and cisplatin were followed for bilateral pulmonary metastases for the next 3 years. During the course, two additional localized radiotherapies were done because the chemotherapy was temporarily stopped due to decreased renal function. Also, one metastatic nodule was surgically removed owing to an unsatisfactory therapeutic response. At his last checkup, there was no evidence of recurrence or metastasis. However, the blood tests showed mild anemia and a slightly increased monocyte proportion (Table 1).

A series of ancillary immunohistochemical stains were done, and as expected, myeloid markers, such as myeloperoxidase (MPO) (Fig. 1G), CD33, and CD68, showed diffuse positivity in the atypical cells. Monocytic markers, such as CD15 (Fig. 1H) and CD4, also showed focal positivity. MPO showed especially strong positivity in all the lesions with infiltration. CD34 and CD117 were positive in a few cells. In contrast, CD3, CD8, CD10, CD20, CD 56, CD79a, CD123, PAX5, and S100 were negative.

As a result, the cause of death was determined to be t-AML with monocytic differentiation (M4 or M5), secondary to adjuvant therapy for his hepatic malignancy, and therefore, the manner of death was natural.

Informed consent was not available because the autopsy was requested by the police and the prosecutor, and the authors could not directly reach to any relatives of the deceased or other qualified individuals.

\section{Discussion}

Usually, an AML may be diagnosed when blasts are greater than $20 \%$ of total WBCs in a PBS or bone marrow biopsy. However, the specific figures do not matter, if there is an extramedullary infiltration of leukemic cells or a molecular change $[2,9]$. This would be useful when bone marrow collection is not available such as a routine medicolegal autopsy. A few researchers reported that postmortem blood smears are not suitable for assessing blood cell quantity or morphology, especially 48 hours following death [10,11]. In the present case, routine histologic examination by blind sampling revealed extensive leukemic infiltrations in the major organs. The blood smear also showed characteristic findings for AML, as presented in Fig. $1 \mathrm{~A}$ and $B$, despite their low quality for interpretation.

The immunohistochemical staining supported the diagnosis of AML in this case. However, this is not a 
common situation in most forensic institutions. The death investigation system is generally the charge of the public and the government. Therefore, the examination tools applicable in a medicolegal autopsy are more restricted than those in clinical processes or hospital autopsies [12]. However, a detailed autopsy diagnosis can help the community to meet their needs. In the present case, the police did not have to find a suspect who might have been responsible for the death, because the deceased died of underlying disease. The family could accept the circumstance of death on this medical basis. This could be utilized in determining

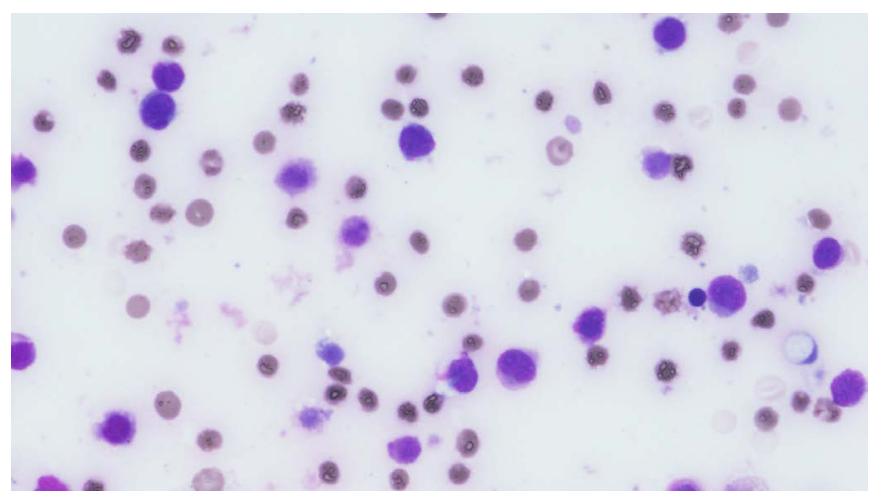

A

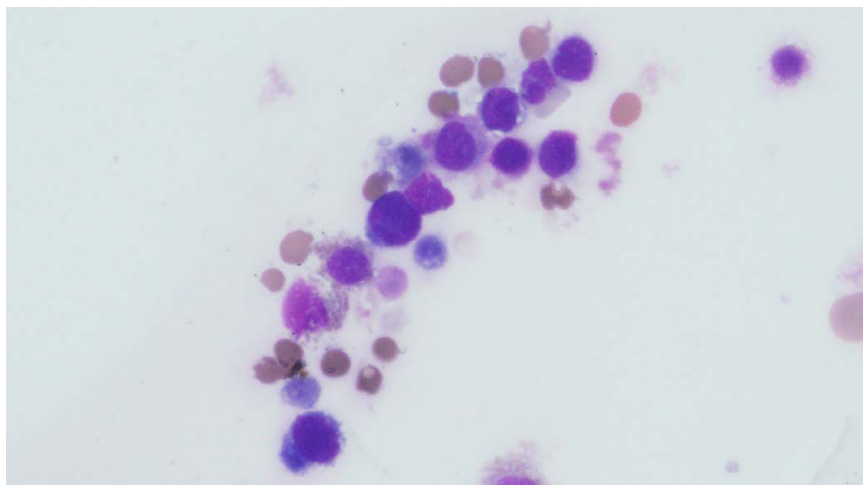

B

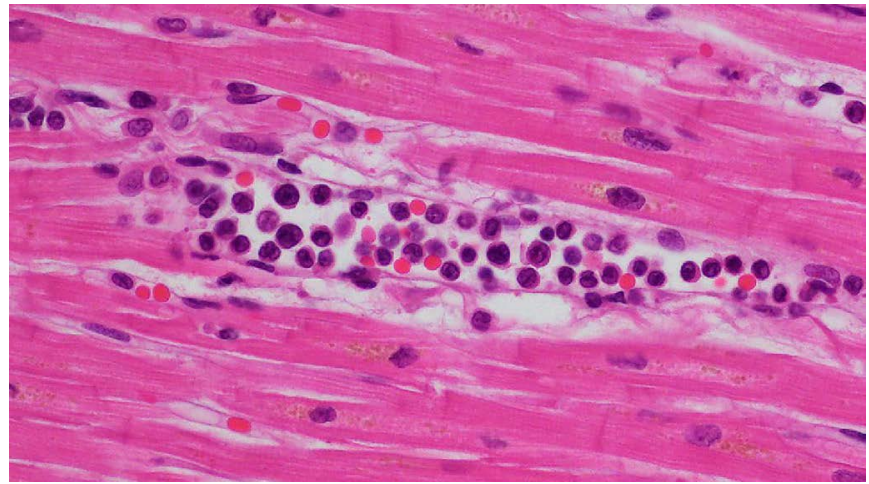

C

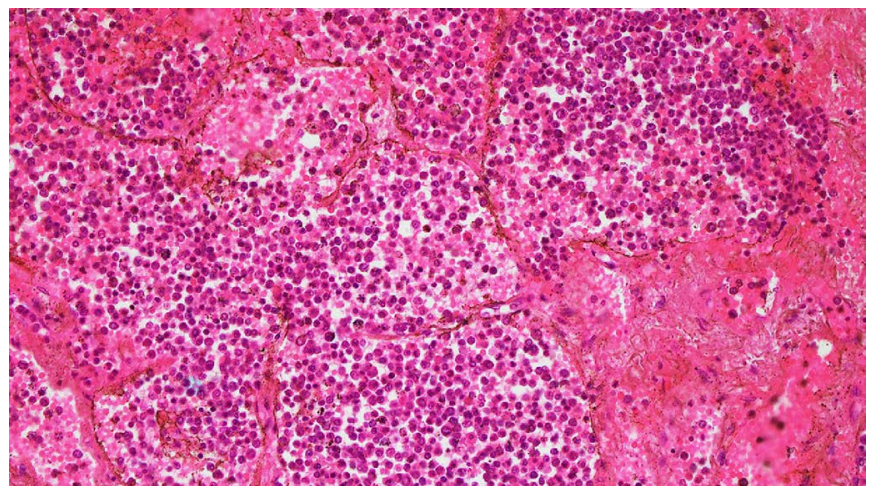

D
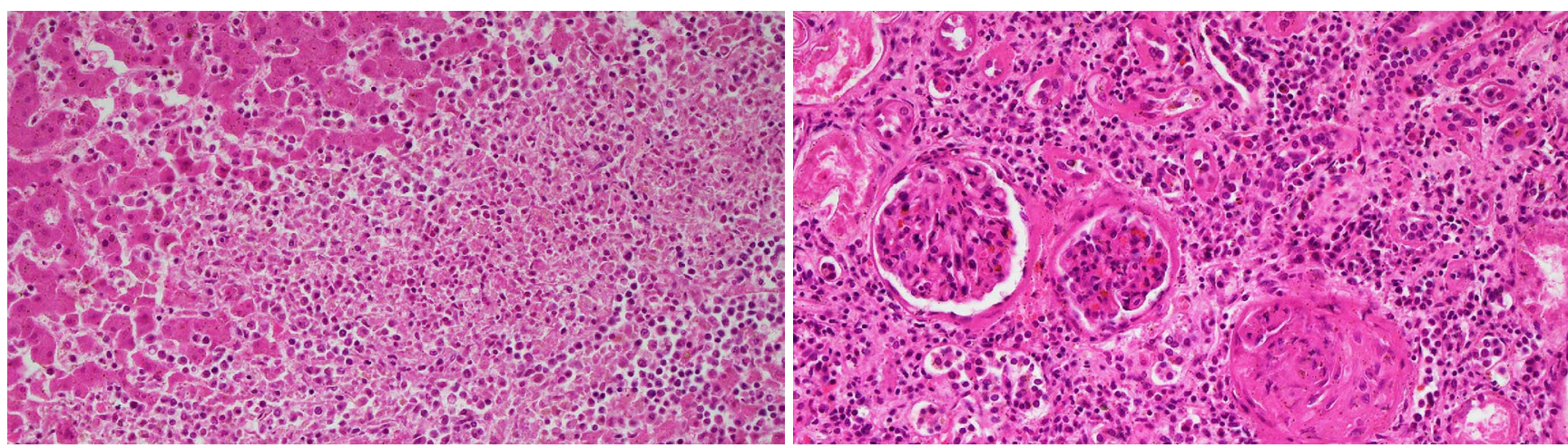

E

$\mathrm{F}$

Fig. 1. Microscopic findings of the major organs of the deceased. In the peripheral blood smear, despite postmortem hemolysis, the proportion of white blood cells was increased $(\mathbf{A}$, Giemsa, $\times 1,000)$ and some blasts could be discerned $(\mathbf{B}, \mathrm{Giemsa}, \times 1,000)$. Leukemic cells were observed in the capillary lumen of the myocardium $(C, H \& E, \times 400)$. In the lung, they filled the alveolar lumen and peribronchial vessels $(D, H \& E, \times 200)$. Focal necrosis of the hepatocytes $(E, H \& E, \times 200)$ induced by the leukemic infiltration were observed in the liver. The microvasculature of the kidney was also infiltrated by the leukemic cells, including the glomerulus $(F, H \& E, \times 200)$. (Continued) 


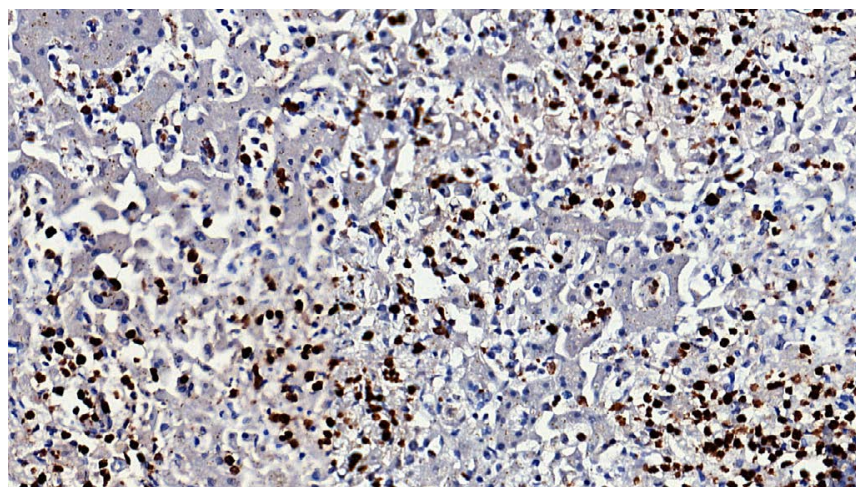

G

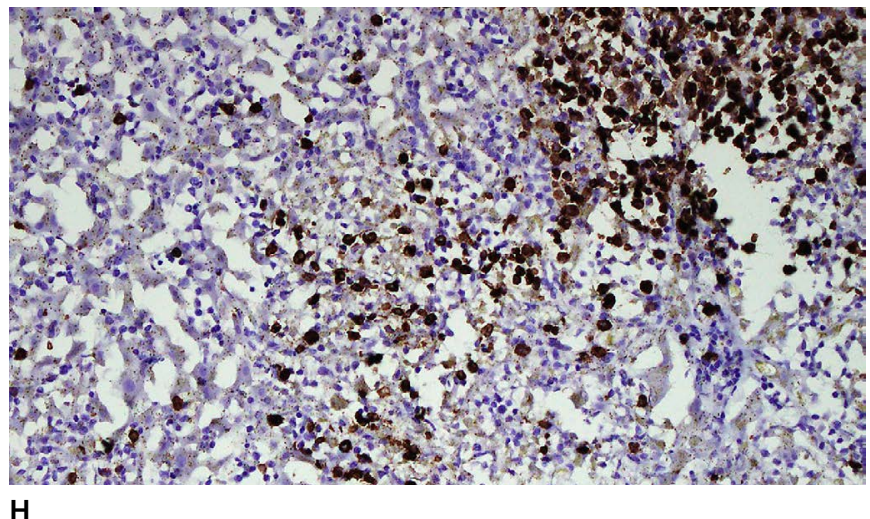

Fig. 1. (Continued) Myeloperoxidase $(\mathbf{G}$, liver, $\times 200)$ and CD15 $(\mathbf{H}$, liver, $\times 200)$ were positive at the neoplastic cells.

death compensation or understanding medical situations outside the hospital.

This case was diagnosed as a t-AML based on the medical history of the deceased. The cytotoxicity of any adjuvant cancer therapy and following DNA damage is believed to be responsible for t-AML $[5,6]$. Variable genetic alterations, such as KMT2A, MLL, and TP53 mutations, and cytogenetic aberrations, such as partial deletion of chromosome 5 or 7 , have been reported to be associated with t-AML or specific prior agents $[5,6]$. However, their role in generating $t-A M L$ or its mechanism are not clear so far. A clinical history is still the most important clue in diagnosing t-AML [4,5], as in the present case. The deceased's chemotherapy regimen consisted of gemcitabine and cisplatin. No genetic mutations or karyotype changes in t-AML have been suggested to be related to these agents.

The liver is not the usual site of primary tumors in patients with t-AML $[4,6-8]$. This seems to be because of the characteristics of hepatic malignancy, which include variety of therapeutic options, low response rate to systemic chemotherapy or radiation, and a poor prognosis at the advanced stage. Systemic chemotherapy or radiation are considered only for unresectable or advanced tumors with an unsatisfactory response to recommended options, such as ablation and embolization. Unfortunately, their outcomes have been generally disappointing, and systemic chemotherapy has recently been replaced by targeted therapy or immunotherapy. However, in the present case, systemic chemotherapy stabilized the growth of metastatic tumors, and the patient had survived 4 years, as long as the known latency period of t-AML $[5,6,8]$. The median survival of an advanced hepatic malignancy is known to be about eight months [13].

AML patients tend to have shorter lifespans than with other malignancies [1]. The patient can collapse immediately following symptom onset or first clinical suspicion. The disease can even be unexpectedly diagnosed after death, as the present case and previous reports show $[9,14]$. Common causes of death in AML patients are major organ failure, infection such as pneumonia, and cerebral hemorrhage [9,14]. Fatal conditions can result from the disease itself or from treatment complications. These include bone marrow failure, hyperleukocytosis (or leukostasis), tumor lysis syndrome, disseminated intravascular coagulation, and chemotoxicity [15].

Among the above conditions, hyperleukocytosis seems to have played a critical role in the present case. This is defined as an excess of WBCs, over 100,000/ $\mathrm{LL}[15,16]$. Leukostasis is a pathologic status of hyperleukocytosis which indicates hypoperfusion in the organ systems on account of intravascular blast accumulation and increased blood viscosity $[16,17]$. Common symptoms are dyspnea, confusion, headache, tinnitus, blurred vision, or focal neurologic deficits [16]. Some rare abnormalities include deep vein thrombosis, splenic rupture, or acute kidney injury [15-18]. Pryds et al. [18] reported a case with right heart failure, which had similar echocardiographic findings to the present case. In the present case, various mechanisms of death could be considered, including respiratory or hepatic failure due to accumulated blasts, an acute heart failure 
as Pryds et al. suggested, or a combination of these conditions.

AML is a systemic disease not treatable with surgery, which implies that diagnosis mainly depends upon blood or small bone marrow samples. In the present autopsy case, the authors could acquire comprehensive gross and microscopic findings of AML progression. This case emphasizes that the hematologic changes of cancer survivors should be carefully observed, and a detailed diagnosis is needed at a medicolegal autopsy to meet social and academic needs.

ORCID: Ki Rim Lee: https://orcid.org/0000-0001-87741204; Jin Ho Paik: https://orcid.org/0000-0002-27920419; Soong Deok Lee: https://orcid.org/0000-00034270-4279; Moon-Young Kim: https://orcid.org/00000002-6381-9377

\section{Conflicts of Interest}

No potential conflict of interest relevant to this article was reported.

\section{References}

1. Shallis RM, Wang R, Davidoff A, et al. Epidemiology of acute myeloid leukemia: recent progress and enduring challenges. Blood Rev 2019;36:70-87.

2. De Kouchkovsky I, Abdul-Hay M. 'Acute myeloid leukemia: a comprehensive review and 2016 update'. Blood Cancer J 2016;6:e441.

3. Hahn A, Giri S, Yaghmour G, et al. Early mortality in acute myeloid leukemia. Leuk Res 2015;39:505-9.

4. Granfeldt Ostgard LS, Medeiros BC, Sengelov H, et al. Epidemiology and clinical significance of secondary and therapyrelated acute myeloid leukemia: a national population-based cohort study.J Clin Oncol 2015;33:3641-9.

5. Higgins A, Shah MV. Genetic and genomic landscape of secondary and therapy-related acute myeloid leukemia. Genes (Basel) 2020;11:749.
6. Kayser S, Dohner K, Krauter J, et al. The impact of therapyrelated acute myeloid leukemia (AML) on outcome in 2853 adult patients with newly diagnosed AML. Blood 2011;117:2137-45.

7. Santo AE, Chacim S, Ferreira I, et al. Effect of therapy-related acute myeloid leukemia on the outcome of patients with acute myeloid leukemia. Oncol Lett 2016;12:262-8.

8. Ornstein MC, Mukherjee S, Mohan S, et al. Predictive factors for latency period and a prognostic model for survival in patients with therapy-related acute myeloid leukemia. Am J Hematol 2014;89:168-73.

9. Van de Louw A, Lewis AM, Yang Z. Autopsy findings in patients with acute myeloid leukemia and non-Hodgkin lymphoma in the modern era: a focus on lung pathology and acute respiratory failure. Ann Hematol 2019;98:119-29.

10. Dokgoz H, Arican N, Elmas I, et al. Comparison of morphological changes in white blood cells after death and in vitro storage of blood for the estimation of postmortem interval. Forensic Sci Int 2001;124:25-31.

11. Berens-Riha N, Sinicina I, Fleischmann E, et al. Comparison of different methods for delayed post-mortem diagnosis of falciparum malaria. Malar J 2009;8:244.

12. Cecchetto G, Bajanowski T, Cecchi R, et al. Back to the future: Part 1. The medico-legal autopsy from ancient civilization to the postgenomic era. Int J Legal Med 2017;131:1069-83.

13. Uchino K, Tateishi R, Shiina S, et al. Hepatocellular carcinoma with extrahepatic metastasis: clinical features and prognostic factors. Cancer 2011;117:4475-83.

14. Dierksen J, Buja LM, Chen L. Clinicopathologic findings of hematological malignancy: a retrospective autopsy study. Ann Clin Lab Sci 2015;45:565-73.

15. Bewersdorf JP, Zeidan AM. Hyperleukocytosis and leukostasis in acute myeloid leukemia: can a better understanding of the underlying molecular pathophysiology lead to novel treatments? Cells 2020;9:2310.

16. Ganzel C, Becker J, Mintz PD, et al. Hyperleukocytosis, leukostasis and leukapheresis: practice management. Blood Rev 2012;26:117-22.

17. Ali AM, Mirrakhimov AE, Abboud CN, et al. Leukostasis in adult acute hyperleukocytic leukemia: a clinician's digest. Hematol Oncol 2016;34:69-78.

18. Pryds K, Rasmussen LE, Andersen NH. Fatal right-sided heart failure due to leukostasis in a patient with leukemic transformation of myelodysplastic syndrome. Clin Case Rep 2019;7:115-9. 